\title{
The need for human identification and the scenario in Nepal
}

The forensic investigation of unidentified bodies has two objectives. The first is to assist for criminal investigations, providing the cause and manner of death and the second is to identify the bodies and, if possible, return them to the family of the dead person. ${ }^{1}$ The latter is essential to formal closure of the loved ones in terms of cultural disposal and legal purposes like settling of the financial issues and on partition shares.

In Nepal, the bodies are kept in the mortuary in refrigerated state, if available till the relatives of the deceased claim them. There is no fixed legal provision or protocol to keep the bodies for certain period of time but some institutes are keeping them for 35 days while others disposing them when the resources become depleted. Although autopsy has to be done at the earliest to prevent the loss of findings important to determine the cause and manner of death. Identification in non-decomposed and intact dead bodies can be easily done by visual examination and affirmation by the relatives once they arrive.

Under the appropriate conditions, visual identification is quite acceptable and does not need to be supplemented with some other form of positive identification. Visual identification is not scientific and can be associated with mistakes. If the examiner is at all unsure about the reliability of a visual identification, some other method of identification should be used. Positive identification entails scientifically establishing identity through the presence of known unique characteristics. Positive methods of identification include fingerprints, footprints, dental characteristics, radiographic comparisons and the presence of certain permanently implanted unique medical devices. $^{2}$

The difficulty in identifying human remains is a global problem. But in our scenario, even intact bodies can't be identified. We are not able to identify the bodies as we have constraints of facilities and adequate efforts and importance is not given in this aspect. Police, human rights workers, media and Forensic experts all have important role in doing so and they all should participate harmoniously in identifying the bodies. In our scenario, the bodies are just kept in refrigerated chamber for a few days passively awaiting the relatives to claim for the bodies. That too, the chambers are not always available at all the centres and those present can't run continuously due to lack of electricity leading to decomposition of the bodies which later become unidentifiable. The trend should be changed and an active search for the missing persons should be done. It can be made possible by constructing ante mortem and post-mortem data and establishment of positive identification.

Whenever an information of missing person is filed in police, the data should be created and circulated to all the centres. And as well, whenever an unidentified body is found, the data regarding the information should be made and this too should be circulated which can be matched with the ante mortem data of the missing person and may lead to positive identification through comparative identification technique. Some comparative data which can be highly useful include; antemortem and post mortem dental radiographs, fingerprints, unique body marks, surgeries, unique number of the prostheses or the implants etc. Even if the positive identification is not made, still the profiling should be done regarding the age, sex, height, race, ethnicity etc. which can narrow the search and sophisticated measures of identification like DNA profiling can be done for the limited number of people.

In our context, whenever a visually identifiable body which otherwise has not been identified is brought for autopsy, the medical officer should take photographs of the whole body as it's brought, face in different views and work along with the police 
who should disseminate the information through different media. The search should be made energetically using the resources to the maximal. The doctors can thus play a vital role in identifying the bodies and contribute towards the humanitarian role towards the society.

\section{Correspondence}

\section{Dr. Nuwadatta Subedi}

Dept of Forensic Medicine and Toxicology,

College of Medical Sciences, Bharatpur, Nepal

Email: drndsubedi@gmail.com

DOI: http://dx.doi.org/10.3126/jemsn.v11i4.14316

\section{REFERENCES}

1. International Committee of the Red Cross. Missing people, DNA analysis and identification of human remains: A guide to best practice in armed conflicts and other situations of armed violence. $2^{\text {nd }}$ Ed. Geneva: ICRC; 2009.

2. Jay Dix, Michael Graham. Time of Death, Decomposition and Identification An Atlas. Florida: CRC Press;2000. 